\title{
Hygroma Kystique Cervical De Découverte Échographique À Lubumbashi : Caractéristique Échographique Et Issue De La Grossesse
}

\author{
Mwilambwe Ngoy Steve ${ }^{1}$, Otshudi Diumu $\mathrm{Jc}^{1}$, Kabila Mfyama $\mathrm{M}^{1}$, Pataule \\ Fatuma $^{1}$, Biayi Mukendi $\mathbf{J}^{1}$, Eric Kassamba ${ }^{2}$, Munkana Ndundula ${ }^{1,3}$ \\ ${ }^{1}$ (Département De Gynécologie Obstétrique, Université De Lubumbashi, République Démocratique Du Congo) \\ ${ }_{2}^{2}$ (Service De Laboratoire, Université De Lubumbashi, République Démocratique Du Congo) \\ ${ }^{3}$ (Service D’imagerie, Université De Lubumbashi, République Démocratique Du Congo)
}

\begin{abstract}
FetalCystic hygroma is a congenital malformation of the lymphatic system. It's characterized by an anechoic cystic mass, which is located in the posterior cervical and on either side of the median line, having at least two cubicles separated by a median wall. The cervical hygroma is seen at 0.3 to $2 \%$ of fetuses, but this impact varies depending on the population studied and gestational age. We report a case observed for the first time in Lubumbashi probably in the Democratic Republic of Congo during a T2 ultrasound in a primigravida 25 years: male fetus with cervical retro mass with 4 stalls, fetal hydrops, peri-ventricular calcifications and nasal bone hypoplasia.
\end{abstract}

Keywords: Hygroma, Lubumbashi, Ultrasound, DRC

\section{Introduction}

Hygroma kystique foetal est une malformation congénitale du système lymphatique. Il se caractérise par une masse kystique anéchogène, cervicale postérieure de part et d'autre de la ligne médiane, comportant au moins deux logettes séparées par une cloison médiane. Il résulte de l'anomalie de développement du réseau lymphatique cervical postérieur s'accompagnant de la séquestration de liquide lymphatique [1]. L'hygroma cervical est constaté chez 0.3 à $2 \%$ des foetus, mais cette incidence varie en fonction de la population étudiée et de l'âge gestationnel. Il est associé dans 50 à $80 \%$ des cas selon les séries à une dyschromosomie en particulier le syndrome de Turner [2]. Le diagnostic est essentiellement échographique et histopathologique. Le caryotype est un examen clef dans l'enquête étiologique. L'objectif était de décrire les éléments échographiques et biologiques d'un cas observé pour la première fois à Lubumbashi au cours d'une échographie de routine (T2) et faire une revue de la littérature.

\section{Patient Et Observation}

Les auteurs rapportent un cas observé au cours d'une échographie T2 chez une primigeste de 25 ans : grosssesse singleton, sexe masculin, actif, présentant une masse retro-cervicale comprenant 4 logettes dont les plus grandes ont mesuré 27,7 et $27,9 \mathrm{~mm}$ de diamètre (Fig.1a), un œdème sous-cutané de $9 \mathrm{~mm}$, deux calcifications péri-ventriculaires, une hydrocéphalie, une ascite, un hydrothorax (Fig.2), l'hypoplasie de l'os propre du nez, l'index du liquide amniotique mesuré à $214 \mathrm{~mm}$, un placenta épais de 79,3 $\mathrm{mm}$, la biométrie correspondait à $17 \mathrm{SA}$ et 3 jours. Nous n'avions pas noté des malformations cardiaques ni de la colonne vertébrale. Il sera donc retenu un hygroma kystique cervical associé à un hydrops foetal, une hypoplasie de l'os propre du nez et des calcifications péri ventriculaires sur une grossesse évolutive de 17 Semaines et 3 jours.

Le bilan réalisé montrait une sérologie Elisa toxoplasme gondü Ig G > $196 \mathrm{UI} / 1$, les autres ; toxoplasme gondü Ig M, Cytomégalovirus Ig G et Ig M, Rubéole Ig G et Ig M, parvovirus B19 Ig G et Ig M, étaient absents. L'enquête dans la famille n'avait pas révélée d'anomalie génétique. Le diabète était exclu par une glycémie normale. Le caryotype n'a pas été réalisé à cause des contraintes techniques. En définitif, il a été retenu un hygroma kystique cervical avec hydrops foetal et une hypoplasie de l'os propre du nez. Une interruption médicale de la grossesse a été réalisée (Fig. 2).

\section{Discussion}

La littérature n'est pas toujours très explicite en ce qui concerne la définition échographique de l'hygroma kystique [3]. Pour certains, la définition de l'hygroma kystique impose la présence de cloisons sagittales ; pour d'autres, cet élément n'est pas indispensable [4]. Robert et al. décrivent deux situations, l'une marquée par la présence d'une masse kystique anéchogène, cervicale postérieure de part et d'autre de la ligne médiane, comportant au moins deux logettes séparées par une cloison médiane. Et l'autre, d'aspect pseudo- 
tumoral, au contenu hétérogène, à paroi épaisse, multicloisonnée [1]. Dans notre cas, il a été constaté une masse kystique anéchogène, cervicale postérieure, comportant 4 logettes (Fig.1a, 1b et 1c).

Pour Robert et al. sa mise en évidence conduit à effectuer un caryotype qui permet de retrouver le plus souvent une monosomie $\mathrm{X}$, mais aussi une trisomie 21 ou 18. En l'absence d'anomalies chromosomiques, l'association à d'autres malformations est possible mais plus rare $(15 \%)$ : malformation cardiaque, hernie diaphragmatique, anomalie squelettique, syndrome d'immobilisme foetal ou syndrome polymalformatif (syndrome de Roberts, de Noonan) [1].

Aly-Abbara décrit 50-80 \% des aneuploïdies associés à l'hygroma kystique cervical. Le plus fréquent est la monosomie $\mathrm{X}$ (syndrome de Turner) avec 65\%, suivi de la trisomie 21, la trisomie 13, trisomie 18, la trisomie 22 , la trisomie 8 , le caryotype $(47, \mathrm{XXY})$ et quinze pourcent d'hygroma kystique ne sont pas associés à une aneuploïdie [2]. D'autres auteurs trouvent le même taux d'aneuploïdie. Cependant, notent des répartitions un peu différentes : taux plus élevé de Trisomie que de monosomie X $[9,10]$. Harold et al. dans une étude observationnelle constatent que deux tiers des hygromas kystiques étaient associés à une aneuploïdie [6]. Dans notre cas, il n'a pas été réalisé un caryotype à cause de l'absence de cet examen dans nos milieux. Néanmoins, il a été constaté une association avec l'hypoplasie de l'os propre du nez et de l'hydrops fotal (Fig. 1d). Signalons que l'hydrops fotal a été fréquemment retrouvé dans $45 \%$ (33 à 75\%) des cas en moyenne avec un très mauvais pronostic $[9,10]$.

Dans son étude comprenant 944 cas d'Hygroma kystique, scholl et al. en 2012, montrent que l'épaisseur de la nuque est strictement corrélé au risque de pronostic défavorable : toute augmentation de $1 \mathrm{~mm}$ de l'Hygroma kystique augmenté de $44 \%$ le risque d'anomalie du caryotype (OR ajusté 1,44), de $26 \%$ le risque d'anomalie congénitale, de $47 \%$ de risque de fausse couche spontanée, mort foetale in utero ou décès néonatal (OR 1,47) et de 77\% le risque global de pronostic défavorable (OR 1,77)[5]

L'hypoplasie des os propres du nez (OPN) au $2^{\mathrm{e}}$ trimestre de la grossesse est un signe positif dans le cadre du dépistage de la trisomie 21, et également si le rapport (BIP/OPN) est supérieur à 11 . Un tiers des foetus trisomiques 21 ont une hypoplasie unilatérale de l'OPN [2]. Dans notre cas, il a été constaté un rapport BIP/OPN à 13 , ceci pourrait faire suspecter une aneuploïdie (trisomie 21 ).

L'évolution de l'hygroma cervical est différente d'un cas à l'autre : Soit vers l'aggravation qui se manifeste par : la formation de volumineux masses pseudokystiques rétro-latérocervicales en forme de ceinture de sauvetage, avec l'extension vers le scalp, et aussi vers les parties latérales et hautes du tronc foetal ; l'anasarque foetoplacentaire par défaut du drainage du liquide lymphatique et qui se manifeste par un œdème généralisé ; un hydrothorax responsable d'une hypoplasie pulmonaire marquée ; un épanchement péricardique et d'une ascite ; puis la modification de la quantité du liquide amniotique qui est souvent diminuée (oligoamnios) [2]. Les mêmes observations ont été retrouvées dans notre cas, sauf l'hydramnios. La mort fotale in-utero survient dans au moins $75 \%$ des cas.

Soit vers la régression spontanée, mais en laissant des séquelles cicatricielles sous cutanées de type de ptérygum coli. Il est néanmoins conseillé d'assurer une surveillance échographique répétée même si l'hygroma a totalement disparu [1]. Jaques S. et al dans une étude menée à Virginia hospital, portant sur les hygromas kystiques congénitaux cervical observent qu'il n'y avait pas de survivants néonatals dans le groupe avec caryotype anormal et que deux à trois pourcent de nouveau-nés étaient normaux lorsque le diagnostic de l'hygroma kystique était fait in utéro [7]. Graesslin et al., constatent que le sex-ratio apparaissait en défaveur des fœtus de sexe féminin et l'issue était défavorable (fausse couche, interruption volontaire, des anomalies structurelles graves) dans $77,7 \%$ des cas (56/72) [8].

Dans notre cas, vu l'inexistence du caryotype et de la présence de l'hypoplasie de l'os propre du nez et de l'hydrops fœtal, il nous a été difficile d'adopter un programme conservateur. Une interruption médicale de la grossesse a été proposée au couple qui donnera son consentement par écrit. Elle a expulsé à 17 semaines d'aménorrhée avec des signes macroscopiques d'une masse retro cervicale (Fig. 2). Un examen anatomopathologique nous a permis de retenir le diagnostic. 


\section{Figures}

Fig. 1a. Coupe transversale montrant une masse kystique cloisonnée de 27,7 et 27,9 mm

Fig. 1b. Coupe transversale montrant deux autres kystes avec cloison épaisse

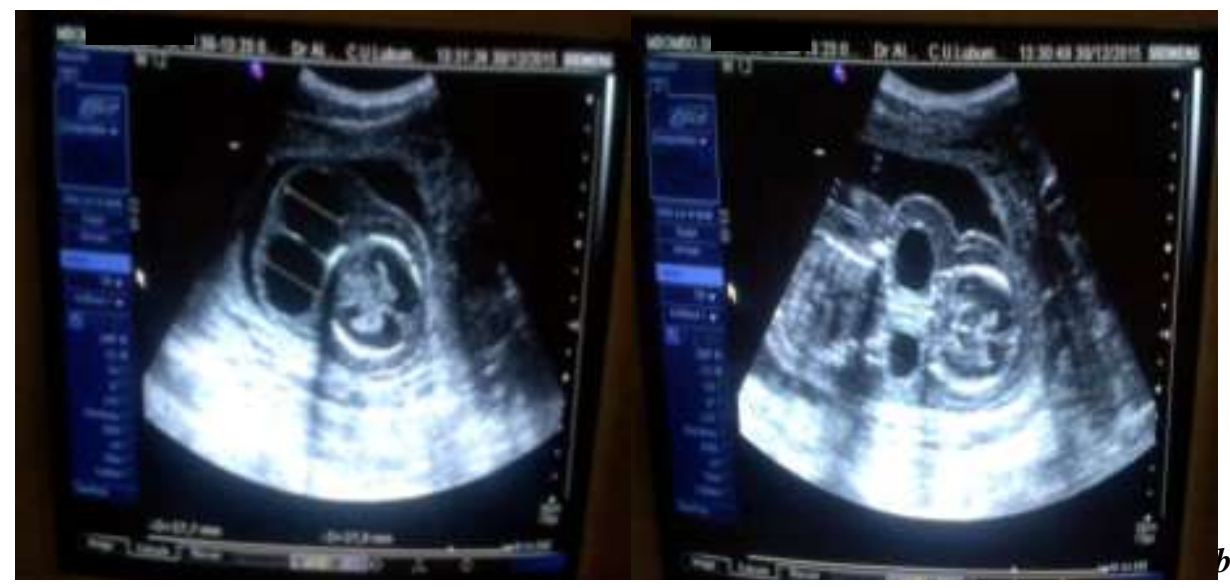

Fig. 1c. Coupe transversale montrant quatre logettes

Fig. 1d. Coupe longitudinale montrant l'hydrothorax et l'ascite
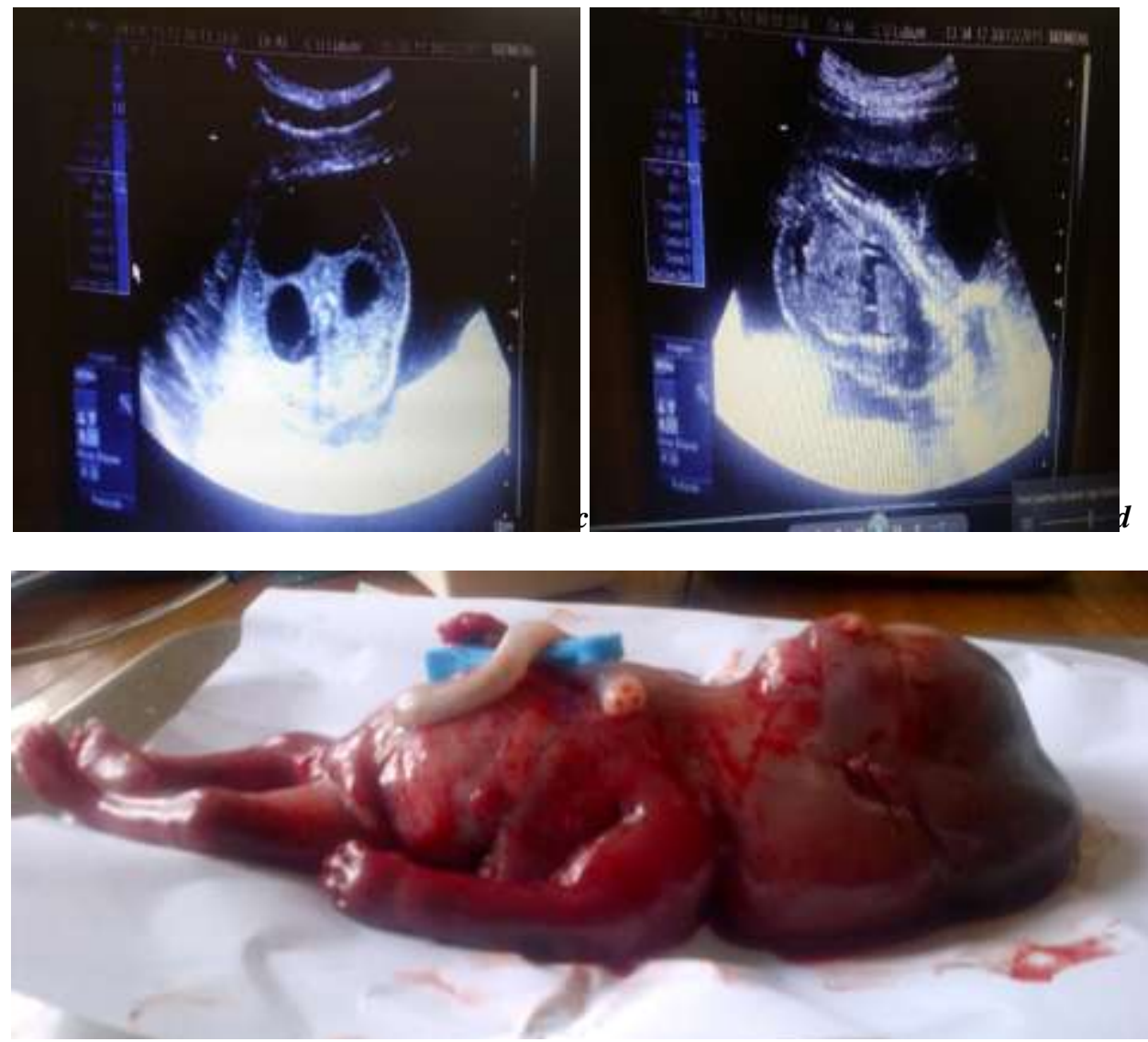

Fig. 2. Nouveau-né avec masse kystique retrocervicale

\section{Conclusion}

L'hygroma kystique est fortement corrélé aux suites périnatales défavorables. Son observation échographique suggère une réalisation du caryotype, ce qui n'est pas d'usage dans notre pays, d'où l'intérêt de rechercher des malformations associées en vue de proposer un canevas de prise en charge propre aux pays à ressources limitées. 


\section{Conflits d'intérêt : Aucun.}

Contribution des auteurs : Tous les auteurs ont participé à la réalisation de ce travail, ils ont lu et approuvé la version finale du manuscrit.

\section{Références}

[1]. Y. Robert, Ph. Bourgeot, B. Guérin, Pathologie cervico-faciale in Bernard Guérin ( $5^{\mathrm{e}}$ Edition) Echographie en pratique obstétricale, (Paris : Elsevier Masson, 2014), p. 257

[2]. Aly-Abbara, Hygroma $\quad$ kystique cervical, http://www.aly-
[int] abbara.com/echographie/Atlas_echographie/images/flash2/hygroma_cervical.html

[3]. C. Lajeunesse, A. Stadler, B. Trombert, M. N. Varlet, H. Patural, F. Prieur, G. Chêne, Hygroma kystique découvert à l'échographie obstétrical du 1er trimestre : caractéristiques échographiques, caryotypiques et de devenir de la grossesse, gynécologie obstétrique et biologie de la reproduction (2014) 43, 455-462

[4]. G. Ducarme, O. Graesslin b, E. Alanio, V. Bige, D. Gaillard, R. Gabriel, Hyperclarté nucale et hygroma cervical au premier trimestre de la grossesse : diagnostic prénatal et devenir néonatal, gynéco obst et fertilité, 2005, vol. 33, $\mathrm{n}^{\circ} 10,750-754$

[5]. Scholl J1, Durfee SM, Russell MA, Heard AJ, Iyer C, Alammari R, Coletta J, Craigo SD, Fuchs KM, D'Alton M, House M, Jennings RW, Ecker J, Panda B, Tanner C, Wolfberg A, Benson CB., First-trimester cystic hygroma: relationship of nuchal translucency thickness and outcomes. Obstet Gynecol. [internet]. 2012, 120 (3):551-9 Available from : http://www.ncbi.nlm.nih.gov/pubmed/ 22872145

[6]. Harold S. Bernstein, Roy A. Filly, James D. Goldberg, Dr. Mitchell S. Golbus, Prognosis of fetuses with a cystic hygroma, prenatal diagnosis (1991), Vol.11, $\mathrm{n}^{\circ} 6,349-355$

[7]. Jacques S. Abramowicz, Steven L. Warsof, Debra Lochner Doyle, Deborah Smith, Donald L. Levy, Congenital cystic hygroma of the neck diagnosed prenatally: Outcome with normal and abnormal karyotype, prenatal diagnosis (1989), vol. 9, $\mathrm{n}^{\circ} 5,321-327$

[8]. Graesslin O, Derniaux E, Alanio E, Gaillard D, Vitry F, Quéreux C, Ducarme G., Characteristics and outcome of fetal cystic hygroma diagnosed in the first trimester. Acta Obstet Gynecol Scand. 2007;86(12):1442-6

[9]. Malone FD, Ball RH, Nyberg DA, Comstock CH, Saade GR, Berkowitz RL, Gross SJ, Dugoff L, Craigo SD, Timor-Tritsch IE, First-trimester septated cystic hygroma: prevalence, natural history, and pediatric outcome. Obstet Gynecol. [internet]. (2005), 106(2):288-94 Available from : http://www.ncbi.nlm.nih.gov/pubmed/

[10]. Tanriverdi HA1, Hendrik HJ, Ertan AK, Axt R, Schmidt W., Hygroma colli cysticum: prenatal diagnosis and prognosis. Am J Perinatol. [internet]. 200118 (8):415-20 Available from : http://www.ncbi.nlm.nih.gov/pubmed/11733855 